\title{
GESTURE CONTROLLED ROBOT USING ADXL335 ACCELEROMETER
}

\author{
Ashish B ${ }^{1}$ \\ ${ }^{1}$ Student, Department of Electronics and Communication, BNM Institute of Technology, Bangalore, Karnataka, India
}

\begin{abstract}
In the field of hostile conditions, space is often considered as the harsh environment at its height and due to the extremities involved. However, it's not necessary to go so far away to find out other situations with such extreme conditions like high temperatures, freezing cold (for example arctic missions), humidity, high pressure level (like in oceanic trench), reduced accessibility and so on and so forth. This paper shows a way how to achieve all of this without risking human life using gesture controlled robots. Then, a robot can be useful to achieve some tasks just impossible to complete by a human operator. It just needs to be controlled by a human thus allowing a human to believe that he is actually there doing that task. It is more importantly used during natural calamities to lift or move heavy rubble to search for survivors. It is also used in scientific research. It is used to gather scientific data from dangerous and hazardous environments like in volcanoes. Wireless controlled robots are very useful in many applications like remote surveillance, military etc. Hand gesture controlled robot can be used by physically challenged in wheelchairs. They are also used to control industrial grade robotic arms. Applications can also include accidentally contaminated scenario (like a nuclear disaster) but also in an intentionally contaminated scenario (like a terrorist act or the use of biologic weapon). In short, the more the environment and the conditions present a threat, the more unmanned specialized robots come on stage. Then the gesture robot is of utmost importance in such scenarios.
\end{abstract}

Keywords: Robot, Gesture, 3- axis Accelerometer and Wireless

$* * *$

\section{INTRODUCTION}

This paper deals with how a robot can be controlled using gesture so that it could be operated in dangerous environments. Gesture recognition is a topic in computer science and language technology with the goal of interpreting human gestures through mathematical algorithms. Gestures can originate from any bodily motion or state but commonly originate from the face or hand. Current focuses in the field include emotion recognition from the face and hand gesture recognition. Many approaches have been made using cameras and computer vision algorithms to interpret sign language. Gesture recognition can be seen as a way for computers to begin to understand human body language. Gesture recognition enables humans to interface with the machine (HMI) and interact naturally without any mechanical devices.

This paper aims to present a robot controlled by gestures which are sensed using an accelerometer. This makes all inaccessible and dangerous environments accessible and feasible due to the low cost involved.

Here, in this robot we have used a AT89S52 microcontroller, RF transmitter, RF receiver, Decoder, Accelerometer, comparator, motot drivers and DC motors.

\section{OVERVIEW}

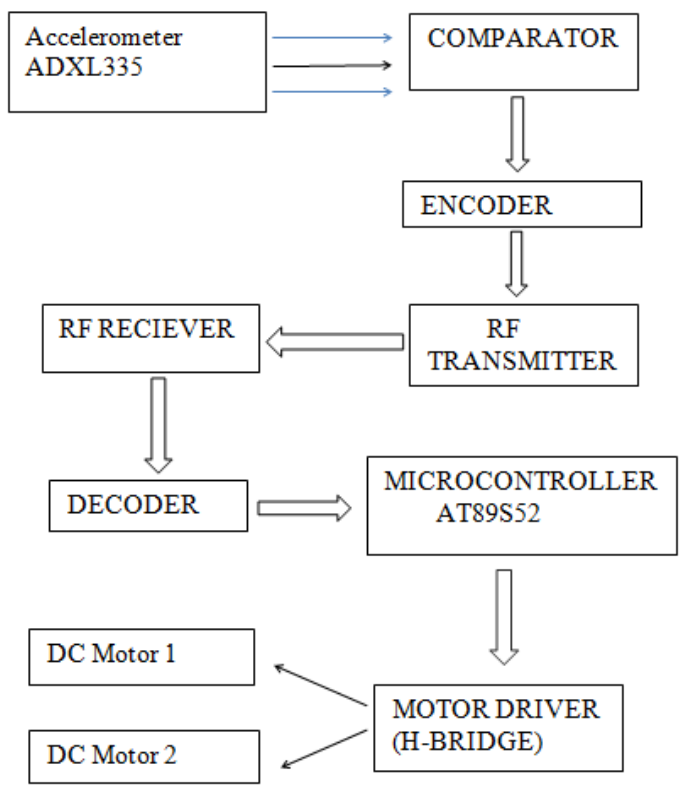

Fig 1: Block diagram of the Gesture controlled robot

\section{COMPARATOR}

It is an operational amplifier, which compares two input voltages and generates a high/low (binary) output.

Comparators are also used as Null detectors, Zero Crossing Detectors, Relaxation Oscillators, Level Shifters, and perhaps the most important application , Analog to Digital Converter (ADC). 
When a comparator performs the function of deciding if an input voltage is above or below a given threshold, it is essentially performing a 1-bit quantization. This function is used in nearly all Analog to Digital Converters (ADC) and hence we use them to generate digital data from sensors' analog voltage input.

In a circuit diagram it is normally represented by a triangle having Inverting (-) and Non-Inverting (+) inputs, Vcc, Ground and Output pins.

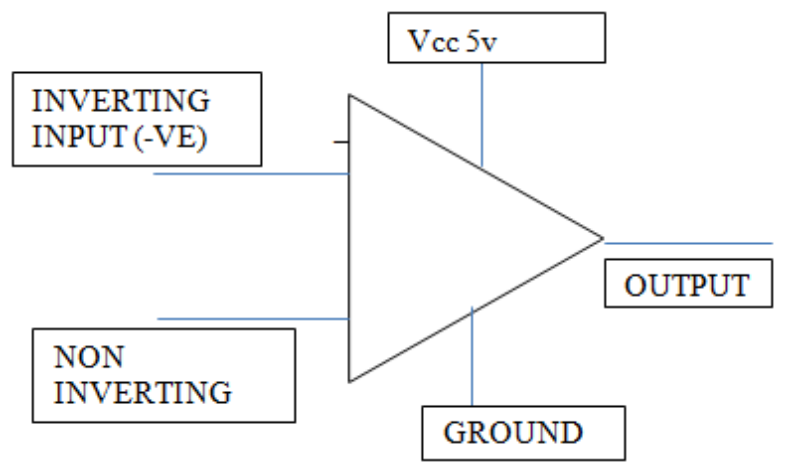

Fig 2: Comparator

\subsection{Operation of a Comparator}

\section{Case 1:}

Assume

Vref(from Potentiometer) is connected to Non-Inverting Input

Vin(sensors' voltage) is connected to Inverting inputthen the output will be as follows:

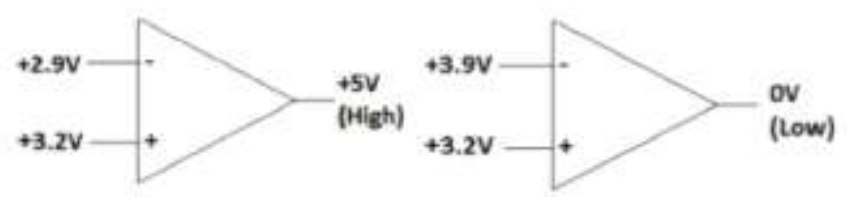

Fig 3: Vin $<$ Vref, output will be high; Vin $>$ Vref output will be low.

Case 2:

Assume

Vref(from Potentiometer) is connected to Inverting Input Vin(sensors' voltage) is connected to Non- Inverting inputthen the output will be as follows:

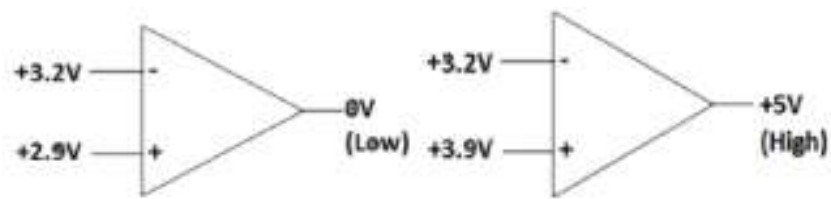

Fig 4: Vin $<$ Vref, output will be low; Vin $>$ Vref, output will be high

From the above observation it is very clear that when sensor Input is connected to the Inverting Input terminal then output changes inversely i.e. for inputs lower than the reference value, higher outputs are obtained and vice versa.
In the robot the comparator works on similar lines. It takes in input from the accelerometer to determine about the direction in which its being tilted. Then this result is fed to the microcontroller.

The comparator used here is LM324, which is a 14 pin IC, consisting of 4 operational amplifiers (inbuilt ). These opamps are operated by a single power supply LM324 and need for a dual supply is eliminated. They can be used as amplifiers, comparators, oscillators, rectifiers etc. But here its only used as comparators.

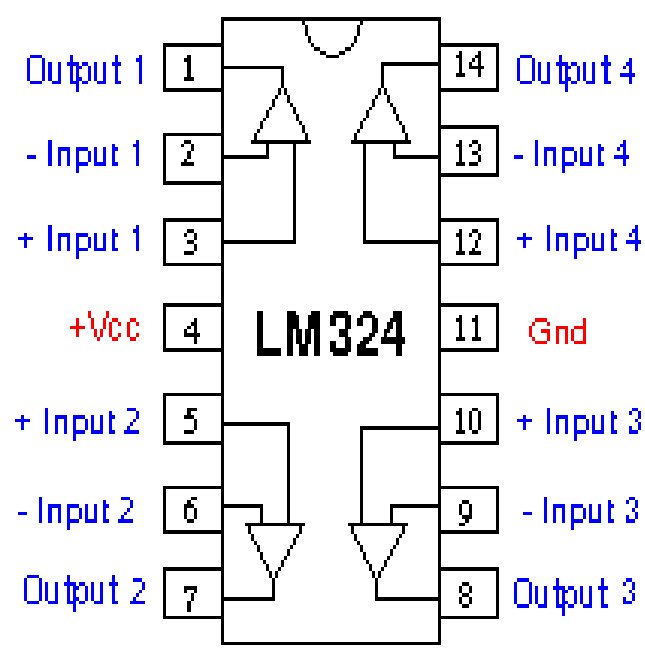

Fig 5: LM324 pin configuration

\section{MICROCONTROLLER}

The Board in use is AT89S52 microcontroller which comes under 8051 family. It consists of $8 \mathrm{kB}$ flash memory and 256 bytes of data RAM.

\subsection{Features of AT89S52}

- $.0 \mathrm{~V}$ to $5.5 \mathrm{~V}$ Operating Range.

- Fully Static Operation: $0 \mathrm{~Hz}$ to $33 \mathrm{MHz}$.

- Three-level Program Memory Lock.

- 256 x 8-bit Internal RAM.

- 32 Programmable I/O Lines.

- Three 16-bit Timer/Counters.

- Eight Interrupt Sources.

- Full Duplex UART Serial Channel.

- Low-power Idle and Power-down Modes.

- Interrupt Recovery from Power-down mode.

- Watchdog Timer.

- Dual Data Pointer.

- Power-off Flag.

- Fast Programming Time.

- Flexible ISP Programming 


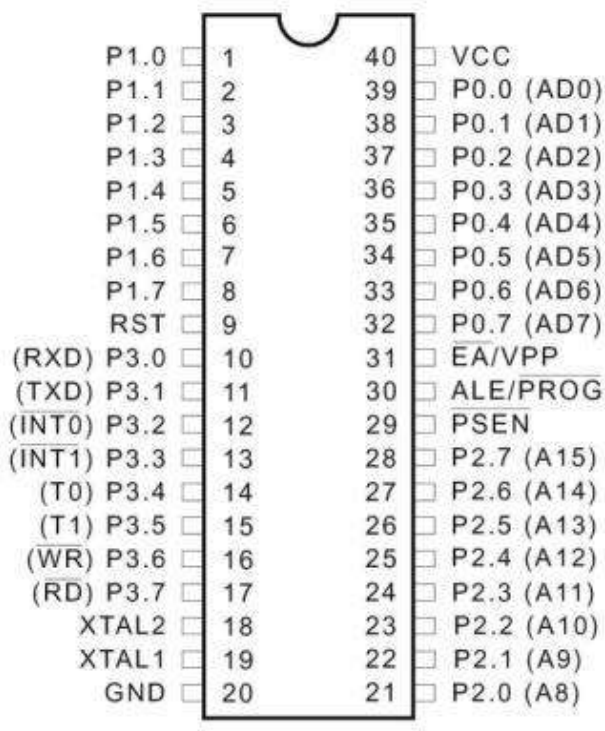

Fig 6: Pin configuration of AT89S52
The AT89S52 is a low-power, high-performance CMOS 8bit microcontroller with $8 \mathrm{~K}$ bytes of in-system programmable Flash memory. The device is manufactured by Atmel

The on-chip Flash allows the program memory to be reprogrammed in-system or by a conventional nonvolatile memory pro-grammer. By combining a 8-bit CPU with insystem programmable Flash on a monolithic chip, the Atmel AT89S52 is a powerful microcontroller which provides a highly-flexible and cost-effective solution to many embedded control applications Considering all these factors, AT89S52 was chosen as the most suitable microcontroller for this robot. This microcontroller was programmed using keil software.

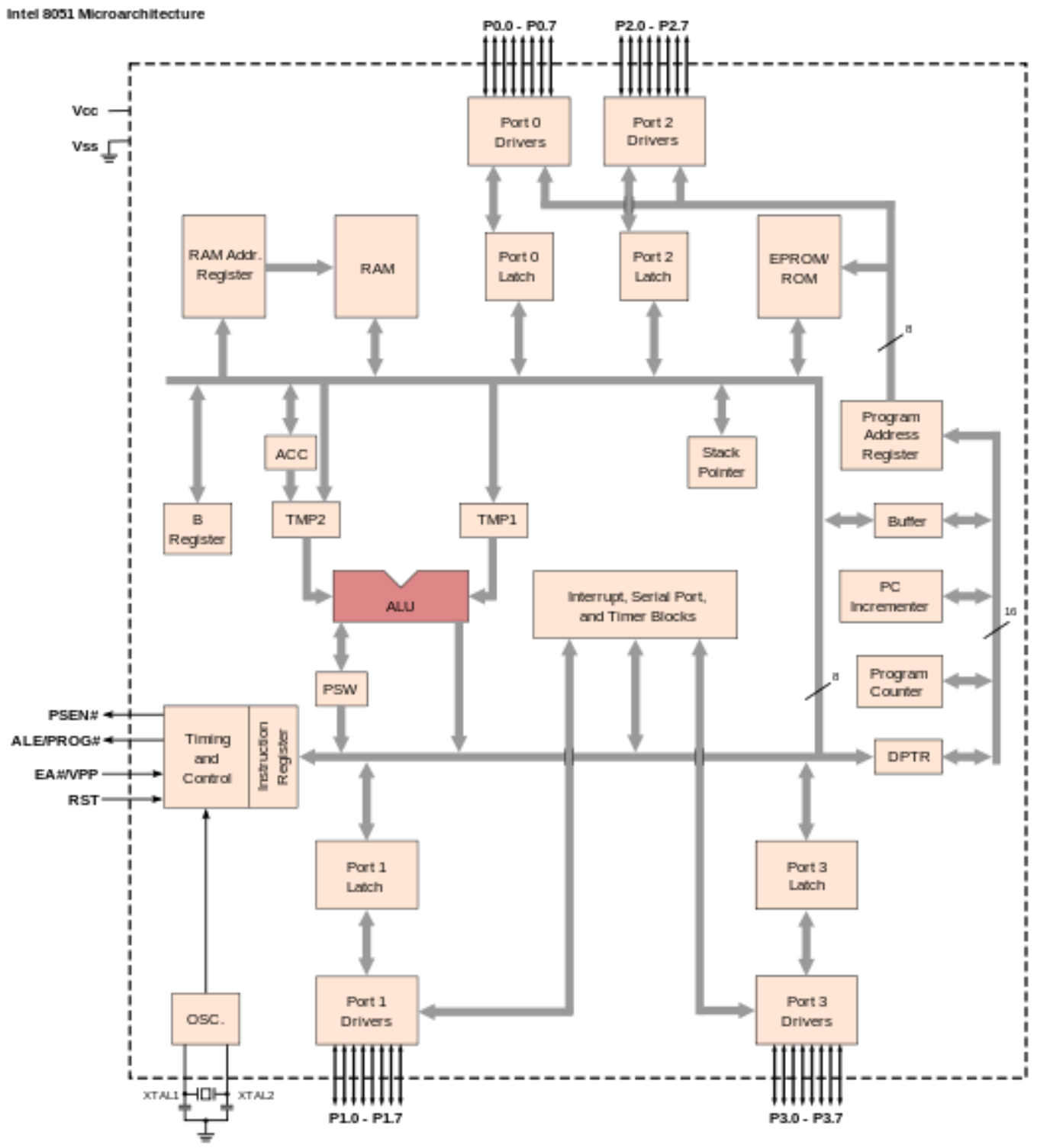

Fig 7: Architecture of AT89S52 


\section{MOTOR-DRIVER}

L293D is a dual H-bridge motor driver integrated circuit (IC). Motor drivers act as current amplifiers since they take a low-current control signal and provide a higher-current signal. This higher current signal is used to drive the motors.L293D contains two inbuilt H-bridge driver circuits. In its common mode of operation, two DC motors can be driven simultaneously, both in forward and reverse direction. The motor operations of two motors can be controlled by input logic at pins $2 \& 7$ and $10 \& 15$. Input logic 00 or 11 will stop the corresponding motor. Logic 01 and 10 will rotate it in clockwise and anticlockwise directions, respectively. Enable pins 1 and 9 (corresponding to the two motors) must be high for motors to start operating. When an enable input is high, the associated driver gets enabled. As a result, the outputs become active and work in phase with their inputs. Similarly, when the enable input is low, that driver is disabled, and their outputs are off and in the high-impedance state.

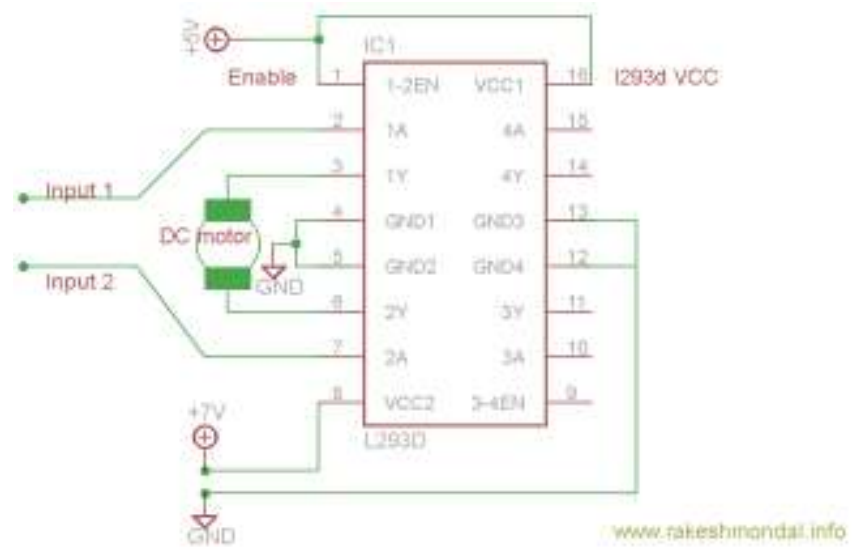

Fig 8: Operation of motor driver L2933D

TRUTH TABLE:

\section{$\underline{A B \quad D E S C R I P T I O N}$}

$0 \quad 0 \quad$ Motor stops or Breaks

01 Motor Runs Anti-Clockwise

10 Motor runs Clockwise

$1 \quad 1 \quad$ Motor stops or Breaks

For the above table, the Enable has to be set 1 .

The above table was used to operate the motor driver using AT89S52 as the microcontroller to send the inputs accordingly. Note that in our robot the PCB is used to drive two motors and the settings need to be done accordingly.

\section{DC MOTORS}

A DC motor belong toa class of electrical machines that converts direct current electrical power into mechanical power. The most common types rely on the forces produced by magnetic fields. Nearly all types of DC motors have some internal mechanism, either electromechanical or electronic, to periodically change the direction of current flow in part of the motor. Most types produce rotary motion; a linear motor directly produces force and motion in a straight line.

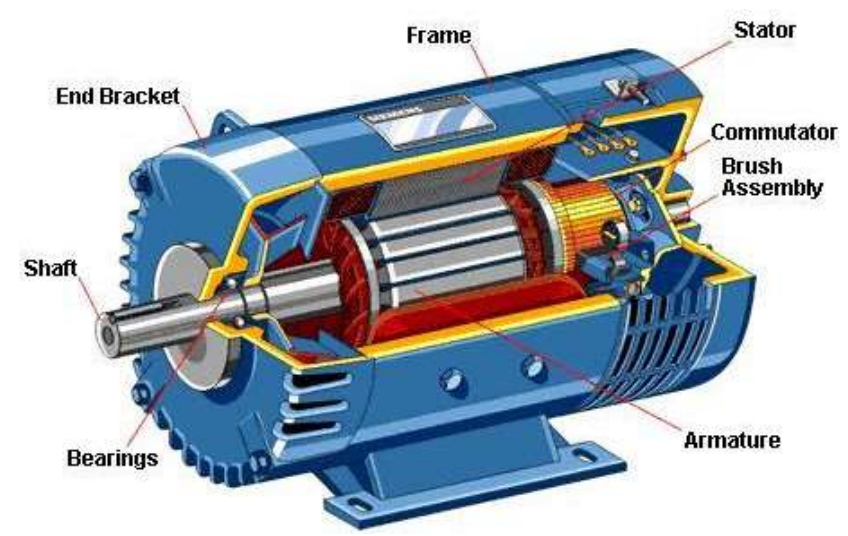

Fig 9: DC motor

D.C Motors are the easiest to control. One D.C Motor requires two signals for its operation. To change its direction one just needs to reverse the polarity of power supply across it.

Speed of motor depends upon its RPM rating and we can vary the speed of motor by changing the voltage across the motor terminals. Gears are used to increase the torque of D.C Motor on the expense of its speed. . Here in our robot we have used 2 robots operated by the motor driver (Hbridge). The truth table mentioned above can be used as a guide to drive the 2 motors.

SPECIFICATIONS OF THE MOTOR USED: 60 RPM-12V DC Geared Motor

\section{ACCELEROMETER}

\subsection{ADXL335}

The ADXL335 is a small, thin, low power, complete 3-axis accelerometer with signal conditioned voltage outputs. The product measures acceleration with a minimum full-scale range of $\pm 3 \mathrm{~g}$. It can measure the static acceleration of gravity in tilt-sensing applications, as well as dynamic acceleration resulting from motion, shock, or vibration. The user selects the bandwidth of the accelerometer using the $\mathrm{CX}, \mathrm{CY}$, and $\mathrm{CZ}$ capacitors at the XOUT, YOUT, and ZOUT pins.

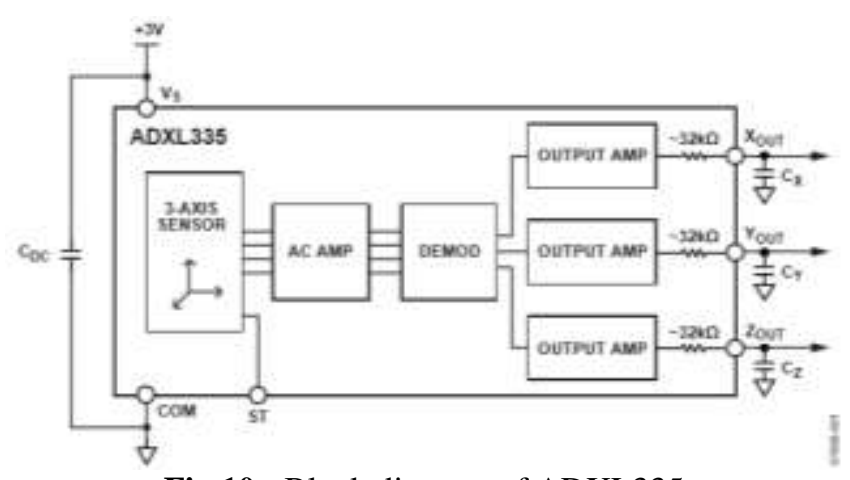

Fig 10: Block diagram of ADXL335 


\subsection{Features of ADXL335:}

- 3-axis sensing

- It can measure the static acceleration of gravity.

- It can also measure dynamic acceleration.

- Temperature stability of ADXL335 is excellent.

- Supply voltage of $5 \mathrm{~V}$ and an operating voltage of $3.3 \mathrm{~V}$

- Has an in-built Voltage Regulator to regulate $5 \mathrm{~V}$ to $3.3 \mathrm{~V}$.

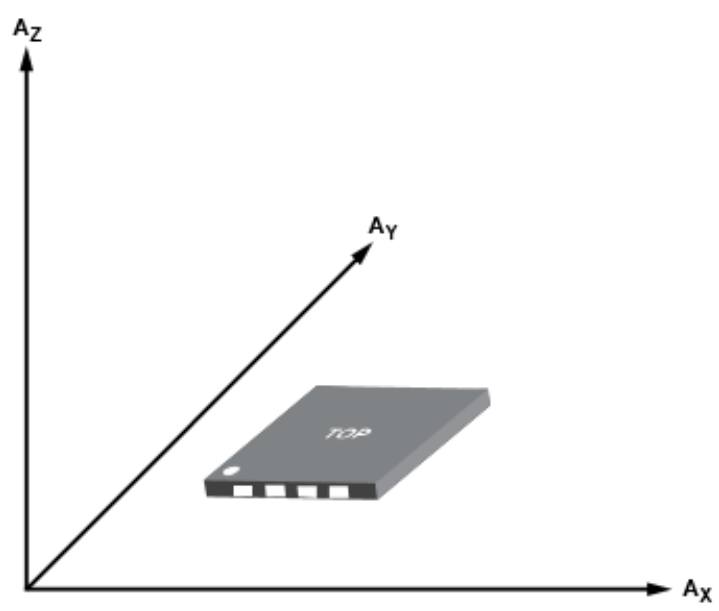

Fig 11: Axes of ADXL335

This accelerometer is used to control the robot, by tilting it sideways or downwards or upwards. It's movement is sensed and the data is transmitted to the microcontroller. The microcontroller is programmed using keil software to control both of the DC motors i.e if the robot has to turn right then DC motor on the right is made to rotate clockwise using the table mentioned above and DC motor on the left is either made to stop or is made to rotate anti-clockwise and vice-versa for the robot to turn left. If the robot has to move forward then both the motors are rotated clockwise. Similarly if it has to move in the reverse direction then the motors are made to rotate anti-clockwise referring the above truth table.

\section{CONCLUSION}

Today we find most robots working for people in industries, factories, warehouses, laboratories, and other places. This paper helps in presenting that robots can also be used in dangerous environments where human cannot venture, especially in space, mining regions, in deep ocean, nuclear power plants etc. Where a lot of risk is involved in sending humans. This paper also helps in proving that robots can be equally efficient in gathering data ,readings and servicing by enabling them to be gesture controlled by humans, Hence engendering a feeling in experts that they are performing the task ( though it's virtual) and the results obtained will be equally accurate with less risk involved. This gesture controlled robot can be made more easier and efficient to operate by installing a high resolution, robust camera with wireless transmission, Thus making this more user friendly.

\section{REFERENCES}

[1]. Muhammad Ali Mazidi, Janice GillispieMazidi, and Rolin D. MaKinlay, "The 8051 Microcontroller And Embedded Systems" Using Assembly and C, Second Edition.

[2]. SencerYeralan\&Ashutosh Ahluwalia, "Programming and interfacing the 8051 Microcontroller"

[3]. Kim Hyoungtae, "How to use Keil 8051 C Compiler"

[4]. David Calcutt, Frederick Cowan, and Hassan Parchizadeh, "8051Microcontroller: An Applications Based Introduction"

[5]. Kenneth Ayala, "The 8051 Microcontroller" third edition.

[6]. Wu X, Su M, Wang P. A hand-gesture-based control interface for a car-robot.IEEE/RSJ International Conference on Intelligent Robots and Systems.Taipei, Taiwan; 2010.

[7]. Raheja JL, Shyam R, Kumar U, Prasad PB. Real-time robotic hand control using hand gestures.Second International Conference on Machine Learning and Computing; 2010.

\section{BIOGRAPHIES}

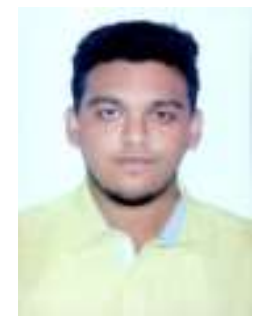

Name: Ashish B ( Born- 1/3/1995)

The author is a student studying in BNM Institute of Technology in electronics and communication branch.

He is worked on many projects including swarm and mobile robotics. He also worked on an IoT based project on which an ieee paper iscurrently being done.

Email :ashishb91@ymail.com

Ph no: +91 9480180174 